\title{
Relationship Between Coupling and the Controllability Grammian in Co-Design Problems
}

\author{
Diane L. Peters ${ }^{\mathrm{a}}$, Panos Y. Papalambros ${ }^{\mathrm{b}}$, A. Galip Ulsoy ${ }^{\mathrm{c}}$ \\ ${ }^{a}$ Corresponding Author: Address: Mechanical Engineering Department, Kettering \\ University, 1700 University Avenue, Flint, MI 48504, USA; phone: 810-762-7916; e-mail \\ dpeters@kettering.edu \\ ${ }^{b}$ Mechanical Engineering Department, University of Michigan, Ann Arbor, MI 48109 \\ USA (e-mail: pyp@umich.edu) \\ ${ }^{c}$ Mechanical Engineering Department, University of Michigan, Ann Arbor, MI 48109 \\ USA (e-mail: ulsoy@umich.edu)
}

\begin{abstract}
Design of smart products requires optimization of both the physical device, or artifact, and its controller. While some components of coupling can be computed a priori, the existence and strength of coupling between these problems over the entire Pareto frontier currently cannot be computed until they are solved. If coupling is expected to be present, then the problem is often solved as a simultaneous, or all-in-one, optimization. This solution process is more difficult, computationally intensive, and operationally inconvenient than a sequential solution method. Consequently, knowing in advance whether coupling is weak or nonexistent is useful.

In this paper, a general formulation for the control problem is given, which includes components for the speed of response, accuracy of the response, and control effort. While this general formulation does not permit a priori determination of coupling, the existence and strength of coupling can be determined for several special cases, which represent common and important control problems. Controllability is a property of the uncontrolled system and does not depend on the controller design. These relationships between coupling and controllability can be utilized in problem formulation, choice of solution method, and development of Control Proxy Functions (CPFs) for each of these problem types. The concepts developed are demonstrated via design of a positioning gantry and a MEMS actuator.
\end{abstract}

Keywords:

Preprint submitted to Mechatronics

March 18, 2015

(C) 2015. This manuscript version is made available under the Elsevier user license http://www.elsevier.com/open-access/userlicense/1.0/ 
co-design, design optimization, coupling, controllability, control proxy function

\section{Introduction}

The development of 'smart' products will solve a wide range of problems in diverse fields. Some applications are structures with active control [1, 2, 3], mechatronics [4, 5, 6, 7], mechanisms [8, 9, 10, 11, 12, 13], microelectrical mechanical systems (MEMS) [14, 15, 16], and chemical processing applications $[17,18]$. These applications include important systems such as robotics [19], aeronautical structures, machine tools, sensors and automotive applications [20, 21]. Such smart products consist of both a physical system, called a plant or artifact, and a controller. To effectively design these products, the possible existence of coupling, or interdependence in the design of the artifact and of the controller, must be considered. Since both uncertainty in system parameters and more demanding performance requirements are associated with more strongly coupled systems [22], the quest for greater efficiency and more robust designs requires better techniques for the identification and management of coupling. Knowledge about coupling provides insight into the nature of the tradeoffs present and guides the choice of an appropriate solution method.

In the optimal design and control, i.e., co-design of these systems, it is necessary to specify one or more objectives for the system. In some cases, a single objective function may adequately capture the systems performance. However, in other cases there are tradeoffs between different system objectives, each of which is critical in the overall system performance. If two objectives are present, with one of them primarily identified with the artifact and the other with the controller, then the problem formulation presented in this paper is appropriate for the system. In the formulation presented here, there are two objective functions. One objective function $f_{a}$ applies to the artifact (e.g., minimize mass), and the other objective $f_{c}$ applies to the controller (e.g., minimize settling time). The full set of variables consists of artifact design variables $\mathbf{d}_{\mathbf{a}}$ and controller design variables $\mathbf{d}_{\mathbf{c}}$. Typically, there are also inequality and equality constraints associated with both the artifact and controller design problems, i.e., $\mathbf{g}_{\mathbf{a}}, \mathbf{h}_{\mathbf{a}}$ and $\mathbf{g}_{\mathbf{c}}, \mathbf{h}_{\mathbf{c}}$, respectively. In the case of bi-directional coupling, both objective functions and sets of constraints depend on both sets of variables. In uni-directional coupling, the artifact objective function and constraints are functions only of the artifact 
variables, while the controller objective function and constraints depend on both artifact and controller variables. In this work, only uni-directional coupling will be addressed. This does exclude many systems; however, there are systems of interest which exhibit uni-directional coupling, and it is a useful starting point for gaining insight into the more general problem.

The co-design problem may be formulated as a combination of the individual objectives. For example, with an objective which is a linear combination, with weights applied to the individual objectives, the co-design problem is formulated as follows:

$$
\min _{\mathbf{d}_{\mathbf{a}}, \mathbf{d}_{\mathbf{c}}} w_{a} f_{a}\left(\mathbf{d}_{\mathbf{a}}\right)+w_{c} f_{c}\left(\mathbf{d}_{\mathbf{a}}, \mathbf{d}_{\mathbf{c}}\right)
$$

subject to

$$
\begin{aligned}
\mathrm{g}_{\mathbf{a}}\left(\mathbf{d}_{\mathbf{a}}\right) & \leq \mathbf{0} \\
\mathbf{h}_{\mathbf{a}}\left(\mathbf{d}_{\mathbf{a}}\right) & =\mathbf{0} \\
\mathrm{g}_{\mathbf{c}}\left(\mathbf{d}_{\mathbf{a}}, \mathbf{d}_{\mathbf{c}}\right) & \leq \mathbf{0} \\
\mathbf{h}_{\mathbf{c}}\left(\mathbf{d}_{\mathbf{a}}, \mathbf{d}_{\mathbf{c}}\right) & =\mathbf{0}
\end{aligned}
$$

Two issues involved in the optimal co-design of coupled systems are: (a) identification of tradeoffs between the two objectives, and (b) selection of appropriate methods of solution. These tradeoffs are present because the optimal design and optimal control problems are coupled, and this coupling cannot be fully quantified prior to solution of the optimization problem. Coupling can be partially computed prior to solution of the full problem, as shown in [23], which can be useful in some cases. In that work, it was shown that coupling can be expressed as a product of two components. One of these components, static gradient terms which capture information about the plant, can be computed a priori, while the second component, the integral of optimal control co-states, cannot. Knowing these static terms, it is possible to identify some cases in which coupling vanishes, i.e., those cases where the static terms vanish. However, this does not provide full information on coupling, which cannot be computed prior to full solution of the problem, since the optimal control co-states are not known prior to solution of the problem.

If coupling is known to exist, then the problem should be solved using a method that lends itself to coupled systems. The solutions found in a simultaneous, or all-in-one, optimization are system-optimal; however, this 
approach is computationally intensive. In strongly coupled systems, an all-inone formulation is indeed preferable, and many advances have been made in this area, improving the computational efficiency significantly. In other systems, however, this approach may not be necessary, if a modified sequential approach can be taken. The modified sequential approach also allows for the use of specialized control techniques, such as root-locus or LQR, which are well established and understood. Furthermore, the simultaneous formulation requires the use of more than one discipline to formulate the full problem. This presents organizational challenges, since expertise in the various disciplines typically resides in different individuals, and often in different groups within an organization. Iterative approaches may also be useful for coupled systems, such as the approach outlined in [23], or the nested optimization method used in [24]. However, these approaches similarly require the full problem to be formulated before solution.

A sequential approach, while easier to solve, does not typically find the system optimum. A modified sequential approach, such as that proposed in $[25,26,27,28]$, may provide system-optimal solutions; however, this method requires the specification of an appropriate Control Proxy Function (CPF). An appropriate CPF needs to capture the fundamental control limitations, and has been shown to be related to the coupling vector $[25,27]$. Thus, in addition to indicating which solution methods might be most appropriate for a given problem, knowledge of coupling can be used to determine what CPF may be used to implement this particular method.

There are other methods and variations on these methods as well, such as those focused on systems where only partial redesign is possible due to system constraints [29]. Other methods focus on nonlinear systems [30, 31]. Yet other methods are based on decomposition-based approaches [32]. New methods are continually being developed, for application to problems with specific characteristics.

In this paper it is shown that for some problem formulations, representing important classical control problems, the existence and strength of coupling can be determined a priori using the controllability Grammian, which offers a significant advantage both in formulating the co-design problem and in choosing appropriate methods of solution. The metrics used for coupling and controllability are introduced in Section 2. Section 3 introduces the positioning gantry system used throughout this paper to illustrate the relationships between coupling and controllability. In Section 4, a general control objective function is given, and then several specific special cases are addressed. 
For these cases, the relationships between coupling and controllability are derived and demonstrated on the positioning gantry system. Section 5 presents an additional case study, the application of the work to a MEMS actuator; two optimizations are carried out for this actuator, one in which coupling is identified and a simultaneous optimization is carried out, and a second one in which knowledge of coupling is used to select a CPF. Finally, Section 6 presents concluding remarks.

\section{Metrics used for Coupling and Controllability}

Several metrics have been developed for quantification of coupling. These metrics include a vector based on optimality conditions [33, 34], a matrix based on the Global Sensitivity Equations (GSEs) [35], and the sensitivities that appear in the GSEs [36]. The metric used here is the vector description of coupling given in Eq. (6), which is applicable to a co-design problem with uni-directional coupling [33]. This metric, derived specifically for problems of this form, is preferred due to its relatively simple form [37].

$$
\Gamma_{\mathbf{v}}=\frac{w_{c}}{w_{a}}\left(\frac{\partial f_{c}\left(\mathbf{d}_{\mathbf{a}}, \mathbf{d}_{\mathbf{c}}\right)}{\partial \mathbf{d}_{\mathbf{a}}}+\frac{\partial f_{c}\left(\mathbf{d}_{\mathbf{a}}, \mathbf{d}_{\mathbf{c}}\right)}{\partial \mathbf{d}_{\mathbf{c}}} \frac{d \mathbf{d}_{\mathbf{c}}}{d \mathbf{d}_{\mathbf{a}}}\right)
$$

where $\boldsymbol{\Gamma}_{\mathbf{v}}$ must be evaluated at the optimal solution to Eqs.(1)-(5). Consequently, the coupling cannot be determined a priori, i.e., before finding a solution to the simultaneous co-design problem in Eqs. (1)-(5). When $\boldsymbol{\Gamma}_{\mathbf{v}}=\mathbf{0}$, the optimization problem is uncoupled. While it is possible for an optimization problem to decouple in cases where $\boldsymbol{\Gamma}_{\mathbf{v}} \neq \mathbf{0}$, i.e., constraint decoupling [24], we will not address that situation in this work; in the cases we consider, decoupling will only occur when $\boldsymbol{\Gamma}_{\mathbf{v}}=\mathbf{0}$. In this case, the artifact design problem

$$
\min _{\mathbf{d}_{\mathbf{a}}} f_{a}\left(\mathbf{d}_{\mathbf{a}}\right)
$$

subject to

$$
\begin{aligned}
& g_{a}\left(d_{a}\right) \leq 0 \\
& h_{a}\left(d_{a}\right)=0
\end{aligned}
$$

can first be solved; then, given the optimal artifact design $\mathbf{d}_{\mathbf{a}}^{*}$, the controller design problem

$$
\min _{\mathbf{d}_{\mathbf{c}}} f_{c}\left(\mathbf{d}_{\mathbf{c}}\right)
$$


subject to

$$
\begin{aligned}
& \mathrm{g}_{\mathrm{c}}\left(\mathrm{d}_{\mathrm{c}}\right) \leq \mathbf{0} \\
& \mathbf{h}_{\mathbf{c}}\left(\mathrm{d}_{\mathrm{c}}\right)=\mathbf{0}
\end{aligned}
$$

can be solved to obtain the same result as obtained from solving the simultaneous co-design problem in Eqs. (1)-(5).

There are several measures of controllability. One metric which is particularly useful in the analysis of co-design problems with coupling is the controllability Grammian matrix, which can be calculated for both constant and time-varying parameter linear dynamical systems. For a system expressed in the form

$$
\begin{aligned}
\dot{\mathbf{x}}(t) & =\mathbf{A} \mathbf{x}(t)+\mathbf{B u}(t) \\
y(t) & =\mathbf{C} \mathbf{x}(t)+\mathbf{D u}(t)
\end{aligned}
$$

the controllability Grammian is the matrix

$$
\mathbf{W}_{\mathbf{c}}(t)=\int_{0}^{t} \boldsymbol{\Phi}(\tau) \mathbf{B B}^{T} \boldsymbol{\Phi}^{T}(\tau) d \tau
$$

where $\boldsymbol{\Phi}(\tau)$ is the state transition matrix [38]. If the matrices $\mathbf{A}$ and $\mathbf{B}$ are time-invariant, then $\mathbf{W}_{\mathbf{c}}(t)$ is given by

$$
\mathbf{W}_{\mathbf{c}}(t)=\int_{0}^{t} e^{\mathbf{A} \tau} \mathbf{B B}^{T} e^{\mathbf{A}^{T} \tau} d \tau
$$

In the case where the final time $t \rightarrow \infty$, the steady-state controllability Grammian, $\mathbf{W}_{\mathbf{c}}^{\infty}$, can also be found by solving the Lyapunov equation

$$
\mathbf{A W}_{\mathbf{c}}{ }^{\infty}+\mathbf{W}_{\mathbf{c}}{ }^{\infty} \mathbf{A}^{T}=-\mathbf{B B}^{T}
$$

The controllability Grammian is often used to determine simply whether or not a system is controllable; if it is singular, the system is not controllable. It can also be used to determine the minimum control effort required to move a system from the origin to a final state $\mathbf{x}_{\mathbf{f}}$ at some final time $t_{f}$, where the control effort, $E\left(t_{f}\right)$, is given by

$$
E\left(t_{f}\right)=\int_{0}^{t_{f}} \mathbf{u}(t)^{T} \mathbf{u}(t) d t
$$




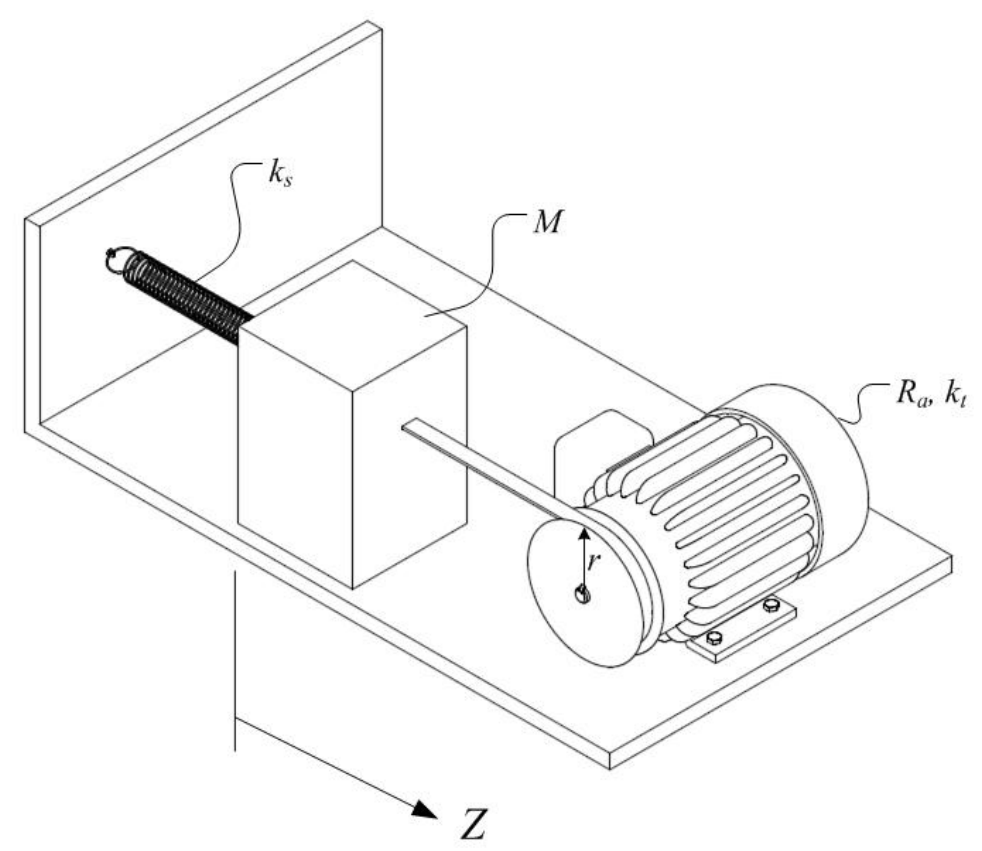

Figure 1: Configuration of Positioning Gantry System

and its minimum value, $E^{*}\left(t_{f}\right)$, is given by [38]:

$$
E^{*}\left(t_{f}\right)=\mathbf{x}_{\mathbf{f}}^{T} \mathbf{W}_{\mathbf{c}}\left(t_{f}\right)^{-1} \mathbf{x}_{\mathbf{f}} .
$$

It is important to note that Eq. (19) is independent of the control structure; it depends only on the dynamics of the uncontrolled system, i.e., $\mathbf{A}$ and $\mathbf{B}$, and the final time $t_{f}$. The optimal (i.e., minimum control) controller performance depends on the controllability Grammian, which is independent of the control architecture and variables. Thus, we will show that, for a large class of important control design problems, the Grammian can be used to determine coupling a priori.

\section{Configuration of Positioning Gantry Example System}

Consider the system shown in Fig. (1), representing a simple model of a positioning gantry. In this system, a mass $M$ is connected to a fixed surface by a linear spring with stiffness $k_{s}$. A belt connects to the mass and wraps around a pulley with radius $r$, which is mounted on a DC motor 


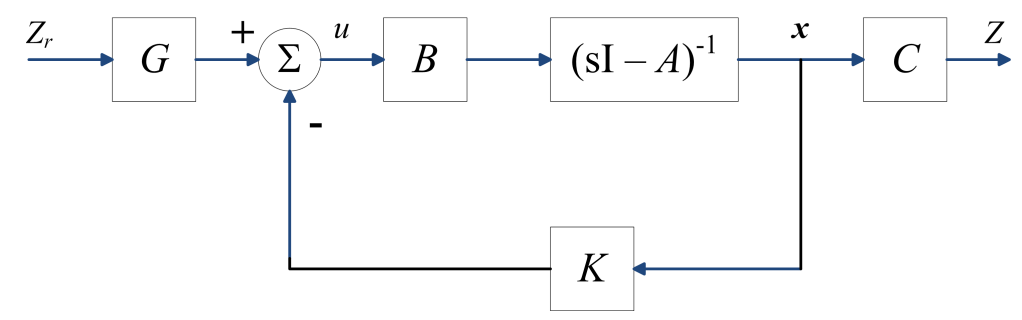

Figure 2: Schematic of System Controller

with armature resistance $R_{a}$ and motor constant $k_{t}$. The mass is assumed to slide on a frictionless surface. The displacement of the mass from its original position is $Z$. The system can be modeled by the following equations:

$$
\begin{aligned}
\dot{\mathbf{x}} & =\mathbf{A} \mathbf{x}+\mathbf{B} u \\
Z & =\mathbf{C} \mathbf{x} \\
\mathbf{x} & =\left[\begin{array}{c}
Z \\
\dot{Z}
\end{array}\right] \\
\mathbf{A} & =\left[\begin{array}{cc}
0 & 1 \\
-\frac{k}{m} & -\frac{b}{m}
\end{array}\right] \\
\mathbf{B} & =\left[\begin{array}{c}
0 \\
\frac{1}{m}
\end{array}\right] \\
\mathbf{C} & =\left[\begin{array}{ll}
1 & 0
\end{array}\right]
\end{aligned}
$$

where $m=\frac{M r R_{a}}{k_{t}}, b=\frac{k_{t}}{r}$, and $k=\frac{k_{s} r R_{a}}{k_{t}}$. A state-feedback controller with gains $\mathbf{K}=\left[\begin{array}{ll}K_{1} & K_{2}\end{array}\right]$ and precompensator $G$ is applied to the system, as shown in Fig. (2), to generate the input voltage $u$ to the motor. The steadystate voltage is denoted as $u_{s s}$. Values of the parameters are $R_{a}=2 \mathrm{k} \Omega$, $M=2 \mathrm{~kg}, k_{s}=2 \mathrm{~N} / \mathrm{mm}$, and $u_{s s}=10 \mathrm{~V}$. The design variables $r$ and $k_{t}$ are found in the optimization of the gantry system using Matlab's fmincon function. This example will be used to illustrate the relationship between coupling and controllability in Section 4. 


\section{Relationships Between $\Gamma_{\mathrm{v}}$ and $\mathrm{W}_{\mathrm{c}}$}

Consider a system whose dynamics are linear and time-invariant, and which is modeled in the form

$$
\begin{array}{r}
\dot{\mathbf{x}}(t)=\mathbf{A}\left(\mathbf{d}_{\mathbf{a}}\right) \mathbf{x}(t)+\mathbf{B}\left(\mathbf{d}_{\mathbf{a}}\right) \mathbf{u}(t) \\
\mathbf{x}(0)=\mathbf{x}_{\mathbf{0}} \\
\mathbf{x}\left(t_{f}\right)=\mathbf{x}_{\mathbf{f}}
\end{array}
$$

It is assumed that this system exhibits uni-directional coupling, as in (1) (5). The objective function for the optimization is a weighted sum of the two individual objectives, where the weights $w_{a}$ and $w_{c}$ are strictly positive.

The control objective function for this system is assumed to take the general form of

$$
f_{c}=\int_{0}^{t_{f}}\left(\mathbf{x}(t)^{T} \mathbf{Q} \mathbf{x}(t)+\mathbf{u}(t)^{T} \mathbf{R} \mathbf{u}(t)+s\right) d t .
$$

This objective function includes the accuracy of the system's response, weighted by the matrix $\mathbf{Q}$; control effort, weighted by the matrix $\mathbf{R}$; and the speed of response, weighted by the scalar $s$. Such an objective can be formulated either for a fixed terminal time problem or for a steady-state problem, by either assigning a finite value to $t_{f}$ or by assuming that $t_{f} \rightarrow \infty$. It is important to note that this formulation is not all-encompassing; there are other terms that could be added, such as energy-based terms, and it could be expanded to include the Mayer (terminal cost) term. However, this formulation does cover a significant range of control objectives that are of interest.

While the existence and strength of coupling has not been derived for this general problem, it is possible to derive useful relationships for several special cases of this general formulation. These special cases represent important classical control problems, and can provide insight into the general problem. The specific cases considered here are described in Table 1.

In the first case, the case of fixed terminal time [39], energy is of primary interest, and the problem is formulated to minimize control effort. In the second case, the speed of response is of importance; a constraint is placed on control effort, but the problem objective is to minimize the response time. In the third case, the control problem is formulated as a classical infinite horizon Linear Quadratic Regulator (LQR) problem, in which a combination of control effort and the system states is minimized. 
Table 1: Summary of Special Cases of General Control Objective Function

\begin{tabular}{|l|l|c|c|c|l|}
\hline & Description & $\mathbf{Q}$ & $\mathbf{R}$ & $s$ & Initial/Final Condition(s) \\
\hline $\begin{array}{l}\text { Case I } \\
\text { Eq. (30) }\end{array}$ & $\begin{array}{l}\text { Minimum Control Effort } \\
\text { with Fixed Terminal Time }\end{array}$ & $\mathbf{0}$ & $\mathbf{I}$ & 0 & $\mathbf{x}_{\mathbf{0}}=\mathbf{0}$ \\
\hline $\begin{array}{l}\text { Case II } \\
\text { Eq. (46) }\end{array}$ & $\begin{array}{l}\text { Minimum Response Time } \\
\text { with Fixed Control Effort }\end{array}$ & $\mathbf{0}$ & $\mathbf{0}$ & 1 & $\mathbf{x}_{\mathbf{0}}=\mathbf{0}$ \\
\hline $\begin{array}{l}\text { Case III } \\
\text { Eq. (59) }\end{array}$ & Infinite Time LQR & $\begin{array}{c}\text { positive } \\
\text { semi- } \\
\text { definite }\end{array}$ & $\begin{array}{l}\text { positive } \\
\text { definite }\end{array}$ & 0 & $\mathbf{x}_{\mathbf{f}}=\mathbf{0}$ \\
\hline
\end{tabular}

\subsection{Case I: Control Effort as Objective}

In Case I, the controller objective function is the control effort required to move the system from the origin to a state $\mathbf{x}_{\mathbf{f}}\left(\mathbf{d}_{\mathbf{a}}\right)$ at some specified time $t_{f}$, where $t_{f}$ is a parameter, as given by Eq. (18). Using (19) and (18), the controller objective function $f_{c}\left(\mathbf{d}_{\mathbf{a}}, \mathbf{d}_{\mathbf{c}}\right)$ will satisfy the relation

$$
f_{c}\left(\mathbf{d}_{\mathbf{a}}, \mathbf{d}_{\mathbf{c}}\right) \geq \mathbf{x}_{\mathbf{f}}\left(\mathbf{d}_{\mathbf{a}}\right)^{T} \mathbf{W}_{\mathbf{c}}\left(\mathbf{d}_{\mathbf{a}}\right)^{-1} \mathbf{x}_{\mathbf{f}}\left(\mathbf{d}_{\mathbf{a}}\right)
$$

where the equality applies if an optimal (i.e., minimum control effort) controller is chosen. The coupling vector is computed from (6) as follows:

$$
\boldsymbol{\Gamma}_{\mathbf{v}}=\frac{w_{c}}{w_{a}}\left[\begin{array}{c}
\boldsymbol{\Gamma}_{\mathbf{v}}=\frac{w_{c}}{w_{a}} \frac{\partial}{\partial \mathbf{d}_{\mathbf{a}}}\left(\mathbf{x}_{\mathbf{f}}\left(\mathbf{d}_{\mathbf{a}}\right)^{T} \mathbf{W}_{\mathbf{c}}\left(\mathbf{d}_{\mathbf{a}}\right)^{-1} \mathbf{x}_{\mathbf{f}}\left(\mathbf{d}_{\mathbf{a}}\right)\right) \\
\mathbf{x}_{\mathbf{f}}\left(\mathbf{d}_{\mathbf{a}}\right)^{T} \frac{\partial \mathbf{W}_{\mathbf{c}}\left(\mathbf{d}_{\mathbf{a}}\right)^{-1}}{\partial d_{a_{1}}} \mathbf{x}_{\mathbf{f}}\left(\mathbf{d}_{\mathbf{a}}\right)+2 \mathbf{x}_{\mathbf{f}}\left(\mathbf{d}_{\mathbf{a}}\right)^{T} \mathbf{W}_{\mathbf{c}}\left(\mathbf{d}_{\mathbf{a}}\right)^{-1} \frac{\partial \mathbf{x}_{\mathbf{f}}\left(\mathbf{d}_{\mathbf{a}}\right)}{\partial d_{a_{1}}} \\
\mathbf{x}_{\mathbf{f}}\left(\mathbf{d}_{\mathbf{a}}\right)^{T} \frac{\partial \mathbf{W}_{\mathbf{c}}\left(\mathbf{d}_{\mathbf{a}}\right)^{-1}}{\partial d_{a_{2}}} \mathbf{x}_{\mathbf{f}}\left(\mathbf{d}_{\mathbf{a}}\right)+2 \mathbf{x}_{\mathbf{f}}\left(\mathbf{d}_{\mathbf{a}}\right)^{T} \mathbf{W}_{\mathbf{c}}\left(\mathbf{d}_{\mathbf{a}}\right)^{-1} \frac{\partial \mathbf{x}_{\mathbf{f}}\left(\mathbf{d}_{\mathbf{a}}\right)}{\partial d_{a_{2}}} \\
\vdots \\
\mathbf{x}_{\mathbf{f}}\left(\mathbf{d}_{\mathbf{a}}\right)^{T} \frac{\partial \mathbf{W}_{\mathbf{c}}\left(\mathbf{d}_{\mathbf{a}}\right)^{-1}}{\partial d_{a_{n}}} \mathbf{x}_{\mathbf{f}}\left(\mathbf{d}_{\mathbf{a}}\right)+2 \mathbf{x}_{\mathbf{f}}\left(\mathbf{d}_{\mathbf{a}}\right)^{T} \mathbf{W}_{\mathbf{c}}\left(\mathbf{d}_{\mathbf{a}}\right)^{-1} \frac{\partial \mathbf{x}_{\mathbf{f}}\left(\mathbf{d}_{\mathbf{a}}\right)}{\partial d_{a_{n}}}
\end{array}\right]^{T}
$$

Given a particular system, it is possible to express the coupling in terms of the artifact design variables $\mathbf{d}_{\mathbf{a}}$, constants, and parameters in the problem. If the $i$ th term in the coupling vector vanishes, then the $i$ th artifact design variable will not participate in the coupling. If all terms in the coupling vector vanish, then the problem is uncoupled. A particular coupling term will vanish under one of two conditions: 
1. The vectors $\mathbf{x}_{\mathbf{f}}\left(\mathbf{d}_{\mathbf{a}}\right)$ and $\left(2 \mathbf{W}_{\mathbf{c}}\left(\mathbf{d}_{\mathbf{a}}\right)^{-1} \frac{\partial \mathbf{x}_{\mathbf{f}}\left(\mathbf{d}_{\mathbf{a}}\right)}{\partial d_{a_{i}}}+\frac{\partial \mathbf{W}_{\mathbf{c}}\left(\mathbf{d}_{\mathbf{a}}\right)^{-1}}{\partial d_{a_{i}}} \mathbf{x}_{\mathbf{f}}\left(\mathbf{d}_{\mathbf{a}}\right)\right)$ are orthogonal.

2. $\frac{\partial \mathbf{x}_{\mathbf{f}}\left(\mathbf{d}_{\mathbf{a}}\right)}{\partial d_{a_{i}}}=-\frac{1}{2} \mathbf{W}_{\mathbf{c}}\left(\mathbf{d}_{\mathbf{a}}\right) \frac{\partial \mathbf{W}_{\mathbf{c}}\left(\mathbf{d}_{\mathbf{a}}\right)^{-1}}{\partial d_{a_{i}}} \mathbf{x}_{\mathbf{f}}\left(\mathbf{d}_{\mathbf{a}}\right)$.

This can occur when the variables $\mathbf{d}_{\mathbf{a}}$ result in changes in the control effort that counteract the effects of the changes in $\mathbf{x}_{\mathbf{f}}$. As an example, an increased $\mathbf{x}_{\mathbf{f}}$ could be associated with a problem configuration with a more efficient use of control effort.

The problem further simplifies if the final state, $\mathbf{x}_{\mathbf{f}}$, is not dependent on $\mathbf{d}_{\mathbf{a}}$. In that case, the expression given in (30) can be simplified to

$$
\boldsymbol{\Gamma}_{\mathbf{v}}=\frac{w_{c}}{w_{a}}\left[\begin{array}{c}
\mathbf{x}_{\mathbf{f}}{ }^{T} \frac{\partial \mathbf{W}_{\mathbf{c}}\left(\mathbf{d}_{\mathbf{a}}\right)^{-1}}{\partial d_{a_{1}}} \mathbf{x}_{\mathbf{f}} \\
\mathbf{x}_{\mathbf{f}}^{T} \frac{\partial \mathbf{W}_{\mathbf{c}}\left(\mathbf{d}_{\mathbf{a}}\right)^{-1}}{\partial d_{a_{2}}} \mathbf{x}_{\mathbf{f}} \\
\vdots \\
\mathbf{x}_{\mathbf{f}}{ }^{T} \frac{\partial \mathbf{W}_{\mathbf{c}}\left(\mathbf{d}_{\mathbf{a}}\right)^{-1}}{\partial d_{a_{n}}} \mathbf{x}_{\mathbf{f}}
\end{array}\right]^{T}
$$

In this situation, the requirement for decoupling that $\boldsymbol{\Gamma}_{\mathbf{v}}=\mathbf{0}$ will be met if $\frac{\partial \mathbf{W}_{\mathbf{c}}\left(\mathbf{d}_{\mathbf{a}}\right)^{-1}}{\partial \mathbf{d}_{\mathbf{a}}}=\mathbf{0}$ for all feasible values of $\mathbf{d}_{\mathbf{a}}$. Of course, it is also possible for the coupling vector to vanish if the vector $\frac{\partial \mathbf{W}_{\mathbf{c}}\left(\mathbf{d}_{\mathbf{a}}\right)^{-1}}{\partial \mathbf{d}_{\mathbf{a}}} \mathbf{x}_{\mathbf{f}}$ is orthogonal to $\mathbf{x}_{\mathbf{f}}$; however, examining the dependence of the controllability Grammian matrix on the artifact design variables will often indicate whether coupling exists, and indicate its strength.

\subsubsection{Positioning Gantry Example for Case I}

For the positioning gantry described in Section 3, the following objectives and constraints are selected:

$$
\begin{gathered}
f_{a}\left(k_{t}, r\right)=-Z_{f}\left(k_{t}, r\right) \\
2.5 \leq r \leq 7.5 \\
5 \leq k_{t} \leq 20
\end{gathered}
$$


where the final displacement $Z_{f}\left(k_{t}, r\right)$ represents the peak displacement, with a $10 \%$ overshoot over the steady-state displacement, $Z_{s s}\left(k_{t}, r\right)$.

$$
Z_{f}=1.1 Z_{s s}=\frac{1.1 u_{s s} k_{t}}{r R_{a} k_{s}}
$$

The controller objective is

$$
f_{c}\left(k_{t}, r, K_{1}, K_{2}, G\right)=E=\int_{0}^{t_{f}}\left(u\left(k_{t}, r, K_{1}, K_{2}, G, t\right)\right)^{2} d t .
$$

In this problem, $\mathbf{Q}=\mathbf{0}, s=0$, and $\mathbf{R}=[1]$. This optimization problem, then, clearly fits the description for a Case I problem. The controllability Grammian $\mathbf{W}_{\mathbf{c}}\left(k_{t}, r, t_{f}\right)$ of this system is given by

$$
\mathbf{W}_{\mathbf{c}}\left(k_{t}, r, t_{f}\right)=\left[\begin{array}{ll}
W_{c_{11}}\left(k_{t}, r, t_{f}\right) & W_{c_{12}}\left(k_{t}, r, t_{f}\right) \\
W_{c_{21}}\left(k_{t}, r, t_{f}\right) & W_{c_{22}}\left(k_{t}, r, t_{f}\right)
\end{array}\right]
$$

where the individual terms are as follows:

$$
\begin{aligned}
W_{c_{11}}\left(k_{t}, r, t_{f}\right)= & \frac{1}{2 b k}-\frac{2 m e^{-\frac{b}{m} t_{f}}}{b\left(4 m k-b^{2}\right)} \\
& +\frac{e^{-\frac{b}{m} t_{f}}}{2 k \sqrt{4 m k-b^{2}}} \sin \left(\sqrt{4 m k-b^{2}} \frac{t_{f}}{m}\right) \\
& +\frac{e^{-\frac{b}{m} t_{f}}}{2 k\left(4 m k-b^{2}\right)} \cos \left(\sqrt{4 m k-b^{2}} \frac{t_{f}}{m}\right) \\
W_{c_{12}}\left(k_{t}, r, t_{f}\right)= & \frac{e^{-\frac{b}{m} t_{f}}}{4 m k-b^{2}}\left(1-\cos \left(\sqrt{4 m k-b^{2}} \frac{t_{f}}{m}\right)\right) \\
W_{c_{21}}\left(k_{t}, r, t_{f}\right)= & \frac{e^{-\frac{b}{m} t_{f}}}{4 m k-b^{2}}\left(1-\cos \left(\sqrt{4 m k-b^{2}} \frac{t_{f}}{m}\right)\right) \\
W_{c_{22}}\left(k_{t}, r, t_{f}\right)= & \frac{1}{2 b m}-\frac{4 k e^{-\frac{b}{m} t_{f}}}{b\left(4 m k-b^{2}\right)} \\
& +\frac{e^{-\frac{b}{m} t_{f}}}{m\left(4 m k-b^{2}\right)^{3 / 2}} \sin \left(\frac{t_{f}}{m} \sqrt{4 m k-b^{2}}\right) \\
& -\frac{b e^{-\frac{b}{m} t_{f}}}{m\left(4 m k-b^{2}\right)} \cos \left(\frac{t_{f}}{m} \sqrt{4 m k-b^{2}}\right)
\end{aligned}
$$




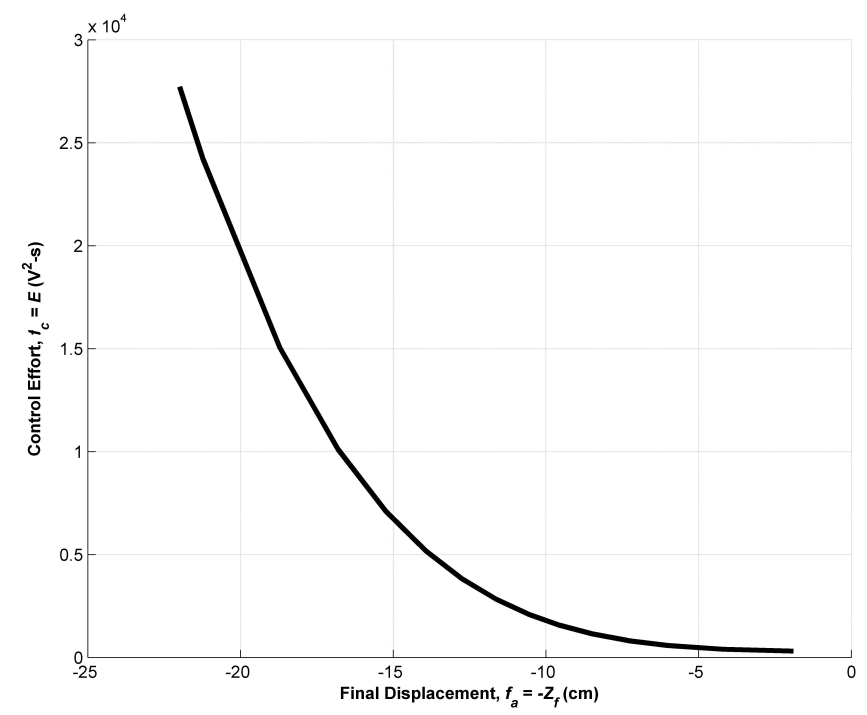

Figure 3: Pareto Frontier for Positioning Gantry Example of Case I

Taking derivatives of both $\mathbf{x}_{\mathbf{f}}\left(\mathbf{d}_{\mathbf{a}}\right)$ and $\mathbf{W}_{\mathbf{c}}\left(\mathbf{d}_{\mathbf{a}}, t_{f}\right)$, it can be shown that there are no feasible values of $r$ and $k_{t}$ for which $\boldsymbol{\Gamma}_{\mathbf{v}}=\mathbf{0}$. Therefore, it is concluded that the problem will be coupled, and an appropriate solution method for a coupled problem should be chosen. When the simultaneous codesign problem is explicitly solved, it is seen to be coupled, with the expected tradeoff between the objectives shown in Fig.(3).

\subsection{Case II: Time as Objective}

In Case II problems, the controller objective function and constraint are as follows:

$$
\begin{aligned}
& f_{c}\left(\mathbf{d}_{\mathbf{a}}, \mathbf{d}_{\mathbf{c}}\right)=t_{f}\left(\mathbf{d}_{\mathbf{a}}, \mathbf{d}_{\mathbf{c}}\right) \\
& \mathbf{g}_{\mathbf{c}}\left(\mathbf{d}_{\mathbf{a}}, \mathbf{d}_{\mathbf{c}}\right)=\int_{0}^{t_{f}} \mathbf{u}\left(\mathbf{d}_{\mathbf{a}}, \mathbf{d}_{\mathbf{c}}, t\right)^{T} \mathbf{u}\left(\mathbf{d}_{\mathbf{a}}, \mathbf{d}_{\mathbf{c}}, t\right) d t-E_{\max } \leq 0
\end{aligned}
$$

Assuming that the constraint is active and that an optimal controller (i.e., one which uses the minimum control effort) is chosen,

$$
\mathbf{x}_{\mathbf{f}}\left(\mathbf{d}_{\mathbf{a}}\right)^{T} \mathbf{W}_{\mathbf{c}}\left(\mathbf{d}_{\mathbf{a}}\right)^{-1} \mathbf{x}_{\mathbf{f}}\left(\mathbf{d}_{\mathbf{a}}\right)=E_{\max } .
$$


Taking derivatives of (44) and solving for $\frac{\partial t_{f}\left(\mathbf{d}_{\mathbf{a}}\right)}{\partial d_{a_{i}}}$,

$$
\frac{\partial t_{f}\left(\mathbf{d}_{\mathbf{a}}\right)}{\partial d_{a_{i}}}=-\frac{2 \mathbf{x}_{\mathbf{f}}\left(\mathbf{d}_{\mathbf{a}}\right)^{T} \mathbf{W}_{\mathbf{c}}\left(\mathbf{d}_{\mathbf{a}}\right)^{-1} \frac{\partial \mathbf{x}_{\mathbf{f}}\left(\mathbf{d}_{\mathbf{a}}\right)}{\partial d_{a_{i}}}+\mathbf{x}_{\mathbf{f}}\left(\mathbf{d}_{\mathbf{a}}\right)^{T} \frac{\partial \mathbf{W}_{\mathbf{c}}\left(\mathbf{d}_{\mathbf{a}}\right)^{-1}}{\partial d_{a_{i}}} \mathbf{x}_{\mathbf{f}}\left(\mathbf{d}_{\mathbf{a}}\right)}{2 \mathbf{x}_{\mathbf{f}}\left(\mathbf{d}_{\mathbf{a}}\right)^{T} \mathbf{W}_{\mathbf{c}}\left(\mathbf{d}_{\mathbf{a}}\right)^{-1} \frac{\partial \mathbf{x}_{\mathbf{f}}\left(\mathbf{d}_{\mathbf{a}}\right)}{\partial t_{f}\left(\mathbf{d}_{\mathbf{a}}\right)}+\mathbf{x}_{\mathbf{f}}\left(\mathbf{d}_{\mathbf{a}}\right)^{T} \frac{\partial \mathbf{W}_{\mathbf{c}}\left(\mathbf{d}_{\mathbf{a}}\right)^{-1}}{\partial t_{f}\left(\mathbf{d}_{\mathbf{a}}\right)} \mathbf{x}_{\mathbf{f}}\left(\mathbf{d}_{\mathbf{a}}\right)}
$$

and the coupling can be expressed as

$$
\boldsymbol{\Gamma}_{\mathbf{v}}=-\frac{w_{c}}{w_{a} D_{N}}\left[\begin{array}{c}
2 \mathbf{x}_{\mathbf{f}}\left(\mathbf{d}_{\mathbf{a}}\right)^{T} \mathbf{W}_{\mathbf{c}}\left(\mathbf{d}_{\mathbf{a}}\right)^{-1} \frac{\partial \mathbf{x}_{\mathbf{f}}\left(\mathbf{d}_{\mathbf{a}}\right)}{\partial d_{a_{1}}}+\mathbf{x}_{\mathbf{f}}\left(\mathbf{d}_{\mathbf{a}}\right)^{T} \frac{\partial \mathbf{W}_{\mathbf{c}}\left(\mathbf{d}_{\mathbf{a}}\right)^{-1}}{\partial d_{a_{1}}} \mathbf{x}_{\mathbf{f}}\left(\mathbf{d}_{\mathbf{a}}\right) \\
2 \mathbf{x}_{\mathbf{f}}\left(\mathbf{d}_{\mathbf{a}}\right)^{T} \mathbf{W}_{\mathbf{c}}\left(\mathbf{d}_{\mathbf{a}}\right)^{-1} \frac{\partial \mathbf{x}_{\mathbf{f}}\left(\mathbf{d}_{\mathbf{a}}\right)}{\partial d_{a_{2}}}+\mathbf{x}_{\mathbf{f}}\left(\mathbf{d}_{\mathbf{a}}\right)^{T} \frac{\partial \mathbf{W}_{\mathbf{c}}\left(\mathbf{d}_{\mathbf{a}}\right)^{-1}}{\partial d_{a_{2}}} \mathbf{x}_{\mathbf{f}}\left(\mathbf{d}_{\mathbf{a}}\right) \\
\vdots \\
2 \mathbf{x}_{\mathbf{f}}\left(\mathbf{d}_{\mathbf{a}}\right)^{T} \mathbf{W}_{\mathbf{c}}\left(\mathbf{d}_{\mathbf{a}}\right)^{-1} \frac{\partial \mathbf{x}_{\mathbf{f}}\left(\mathbf{d}_{\mathbf{a}}\right)}{\partial d_{a_{n}}}+\mathbf{x}_{\mathbf{f}}\left(\mathbf{d}_{\mathbf{a}}\right)^{T} \frac{\partial \mathbf{W}_{\mathbf{c}}\left(\mathbf{d}_{\mathbf{a}}\right)^{-1}}{\partial d_{a_{n}}} \mathbf{x}_{\mathbf{f}}\left(\mathbf{d}_{\mathbf{a}}\right)
\end{array}\right]^{T}
$$

where

$$
D_{N}=2 \mathbf{x}_{\mathbf{f}}\left(\mathbf{d}_{\mathbf{a}}\right)^{T} \mathbf{W}_{\mathbf{c}}\left(\mathbf{d}_{\mathbf{a}}\right)^{-1} \frac{\partial \mathbf{x}_{\mathbf{f}}\left(\mathbf{d}_{\mathbf{a}}\right)}{\partial t_{f}\left(\mathbf{d}_{\mathbf{a}}\right)}+\mathbf{x}_{\mathbf{f}}\left(\mathbf{d}_{\mathbf{a}}\right)^{T} \frac{\partial \mathbf{W}_{\mathbf{c}}\left(\mathbf{d}_{\mathbf{a}}\right)^{-1}}{\partial t_{f}\left(\mathbf{d}_{\mathbf{a}}\right)} \mathbf{x}_{\mathbf{f}}\left(\mathbf{d}_{\mathbf{a}}\right)
$$

Note that the coupling vector is parallel to that seen for Case I, and the conditions for decoupling in this problem are mathematically identical. This indicates that the physical conditions under which the problems decouple are also the same. As in Case I, therefore, one situation which would result in decoupling is that in which changes in $\mathbf{d}_{\mathbf{a}}$ produce both a greater displacement $\mathbf{x}_{\mathbf{f}}$ of the system and a more efficient use of the available control effort. Also as in Case I, if the final state $\mathbf{x}_{\mathbf{f}}$ is a parameter rather than being a function of $\mathbf{d}_{\mathbf{a}}$, then the coupling vector will simplify. In this case, the coupling can 
be expressed as

$$
\boldsymbol{\Gamma}_{\mathbf{v}}=-\frac{w_{c}}{w_{a}\left(\mathbf{x}_{\mathbf{f}}\left(\mathbf{d}_{\mathbf{a}}\right)^{T} \frac{\partial \mathbf{W}_{\mathbf{c}}\left(\mathbf{d}_{\mathbf{a}}\right)^{-1}}{\partial t_{f}\left(\mathbf{d}_{\mathbf{a}}\right)} \mathbf{x}_{\mathbf{f}}\left(\mathbf{d}_{\mathbf{a}}\right)\right)}\left[\begin{array}{c}
\mathbf{x}_{\mathbf{f}}\left(\mathbf{d}_{\mathbf{a}}\right)^{T} \frac{\partial \mathbf{W}_{\mathbf{c}}\left(\mathbf{d}_{\mathbf{a}}\right)^{-1}}{\partial d_{a_{1}}} \mathbf{x}_{\mathbf{f}}\left(\mathbf{d}_{\mathbf{a}}\right) \\
\mathbf{x}_{\mathbf{f}}\left(\mathbf{d}_{\mathbf{a}}\right)^{T} \frac{\partial \mathbf{W}_{\mathbf{c}}\left(\mathbf{d}_{\mathbf{a}}\right)^{-1}}{\partial d_{a_{2}}} \mathbf{x}_{\mathbf{f}}\left(\mathbf{d}_{\mathbf{a}}\right) \\
\vdots \\
\mathbf{x}_{\mathbf{f}}\left(\mathbf{d}_{\mathbf{a}}\right)^{T} \frac{\partial \mathbf{W}_{\mathbf{c}}\left(\mathbf{d}_{\mathbf{a}}\right)^{-1}}{\partial d_{a_{n}}} \mathbf{x}_{\mathbf{f}}\left(\mathbf{d}_{\mathbf{a}}\right)
\end{array}\right]^{T}
$$

which is parallel to the simplified coupling vector given by Eq. (31), with identical conditions for decoupling.

\subsubsection{Positioning Gantry Example for Case II}

Assume, in this case, that the artifact objective and constraints are as given in Eqs. (32) - (34). The control objective and constraints are as follows:

$$
\begin{aligned}
f_{c}\left(k_{t}, r, K_{1}, K_{2}, G\right) & =t_{f}\left(k_{t}, r, K_{1}, K_{2}, G\right) \\
g_{1}\left(k_{t}, r, K_{1}, K_{2}, G\right) & =\int_{0}^{t_{f}}\left(u\left(t, k_{t}, r, K_{1}, K_{2}, G\right)\right)^{2} d t-E^{*} \\
& =E-E^{*} \leq 0
\end{aligned}
$$

Monotonicity analysis indicates that the constraint $g_{1}$ will be active [40]; thus, this problem meets the conditions set down for Case II. The controllability Grammian is given by Eqs. (37)-(41). In this case, the coupling is again non-zero for every allowed value of $r$ and $k_{t}$. When the problem is solved, the anticipated tradeoff between $f_{a}$ and $f_{c}$ is evident, as shown in Fig. (4).

\subsection{Case III: Infinite Horizon Linear Quadratic Regulator (LQR)}

The infinite-time LQR problem is designed to find the optimal control signal $\mathbf{u}(t)$ to transition a system from an initial state $\mathbf{x}_{\mathbf{0}}=\mathbf{x}(0)$ to the zero state. The optimal control signal is defined as the control signal which minimizes the quadratic cost function

$$
f_{c}=\int_{0}^{\infty}\left(\mathbf{x}(t)^{T} \mathbf{Q} \mathbf{x}(t)+\mathbf{u}(t)^{T} \mathbf{R u}(t)\right) d t
$$

In the most general LQR problem formulation, there may also be a crossterm present, i.e., the cost function would include a term $2 \mathbf{x}(t)^{T} \mathbf{S u}(t)$; an 


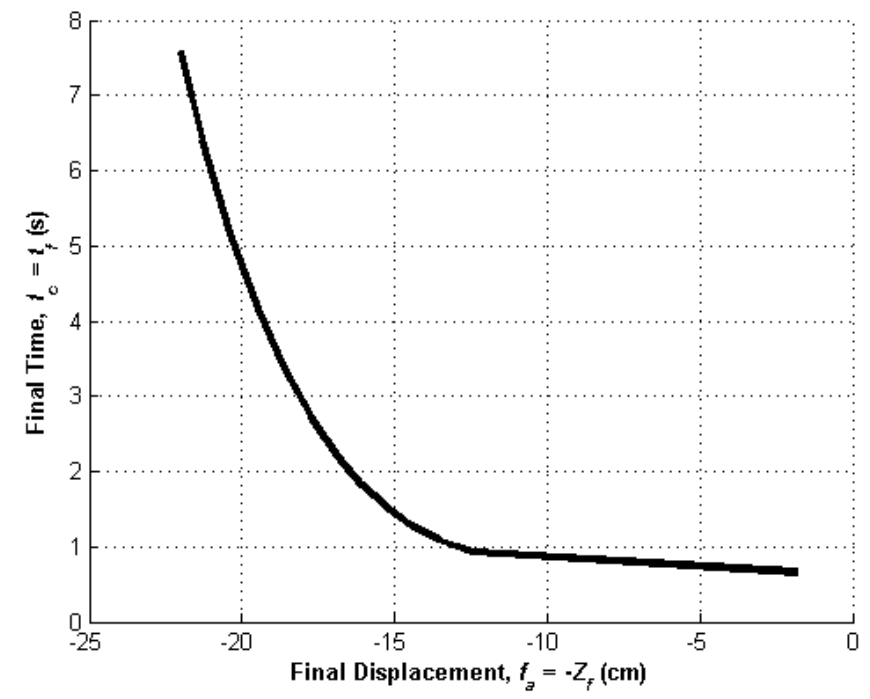

Figure 4: Pareto Frontier for Positioning Gantry Example of Case II

example of this can be seen in [28]. However, it has been shown that a change in variables will reduce that more general problem to the form shown in Eq. (51).

It is well-established [38] that the optimal solution to this LQR problem is

$$
\mathbf{u}(t)=-\mathbf{K} \mathbf{x}(t)
$$

where $\mathbf{K}$ is uniquely determined given $\mathbf{A}, \mathbf{B}, \mathbf{Q}$, and $\mathbf{R}$, and the terms $\mathbf{u}, \mathbf{K}$, and $\mathbf{x}$ have dimensionality of $(m \times 1),(m \times n)$, and $(n \times 1)$, respectively. The LQR problem can be analyzed for the two cases where $m=n$ and $m \neq n$, both of which lead to the same conclusion regarding the relationship between coupling and controllability.

In the case where $m=n$, the gain matrix $\mathbf{K}$ is invertible, and it is possible 
to solve for $\mathbf{x}$ as $\mathbf{x}=-\mathbf{K}^{-1} \mathbf{u}$, and the LQR objective, $J$, can be rewritten as

$$
\begin{aligned}
J & =\int_{0}^{\infty}\left(\mathbf{u}^{T}\left(\mathbf{R}+\left(\mathbf{K}^{-1}\right)^{T} \mathbf{Q}\left(\mathbf{K}^{-1}\right)\right) \mathbf{u}\right) d t \\
& =\int_{0}^{\infty}\left(\mathbf{u}^{T} \mathbf{P u}\right) d t
\end{aligned}
$$

This is in the same form as Eq. (18), with the addition of the matrix $\mathbf{P}$ and the condition that $t_{f} \rightarrow \infty$. The minimum value of $J$, then, will be a function of $\mathbf{W}_{\mathbf{c}}^{\infty}$.

In the case where $m \neq n$, the Moore-Penrose pseudoinverse can be used to write $\mathbf{x}$ in the form of $\mathbf{x}=-\mathbf{K}^{\dagger} \mathbf{u}$, and $\mathbf{P}=\mathbf{R}+\left(\mathbf{K}^{\dagger}\right)^{T} \mathbf{Q}\left(\mathbf{K}^{\dagger}\right)$. This allows an approximation for $J$ to be written as

$$
J \approx \int_{0}^{\infty}\left(\mathbf{u}^{T} \mathbf{P u}\right) d t
$$

and, as in the case of $m=n$, this is in the same form as Eq. (18) and the minimum value of $J$ will be a function of $\mathbf{W}_{\mathbf{c}}^{\infty}$. We can state, therefore, that the LQR objective is a linear function of the infinite-time controllability Grammian when LQR control is used, and can be written as

$$
f_{c}^{*}=\mathbf{x}_{\mathbf{0}}{ }^{T} \mathbf{W}_{\mathbf{c}}^{\infty-1} \mathbf{x}_{\mathbf{0}}+\int_{0}^{\infty}\left(\mathbf{u}^{T}(\mathbf{P}-\mathbf{I}) \mathbf{u}\right) d t
$$

where $\mathbf{u}$ is given by the following relation: [38]

$$
\mathbf{u}(t)=\lim _{t_{f} \rightarrow \infty}\left\{-\mathbf{B}^{T} e^{\mathbf{A}^{T}\left(t_{f}-t\right)} \mathbf{W}_{\mathbf{c}}\left(t_{f}\right)^{-1}\left(e^{\mathbf{A} t_{f}} \mathbf{x}_{\mathbf{0}}\right)\right\}
$$

Since the steady-state control signal is zero, Eq. (56) simplifies to

$$
f_{c}^{*}=\mathbf{x}_{\mathbf{0}}{ }^{T} \mathbf{W}_{\mathbf{c}}^{\infty-1} \mathbf{x}_{\mathbf{0}}
$$

This can then be differentiated to obtain the coupling relation, which is given by

$$
\boldsymbol{\Gamma}_{\mathbf{v}}=\frac{\partial}{\partial \mathbf{d}_{\mathbf{a}}}\left(\mathbf{x}_{\mathbf{0}}^{T} \mathbf{W}_{\mathbf{c}}^{\infty-1} \mathbf{x}_{\mathbf{0}}\right)
$$

This relation can be further simplified, in the case of loop transfer recovery, as shown in [41]. In both that special case and in this more general case, however, the relationship between coupling and controllability is clearly seen. 


\subsubsection{Positioning Gantry Example for Case III}

Consider, again, the positioning gantry system shown in Section II. In this case, the artifact objective function is assumed to be the system's total weight, which takes the specific form

$$
f_{a}\left(r, k_{t}\right)=c_{1}+c_{2} k_{t}^{1.5}+c_{3} r^{2}
$$

where $c_{1}=10, c_{2}=5$, and $c_{3}=2.5$, subject to the bounds given in Eqs. (33) - (34). The controller optimization problem is formulated as an LQR problem with controller objective $f_{c}\left(r, k_{t}, G, K_{1}, K_{2}\right)$, given by Eq. (51) with

$\mathbf{x}_{\mathbf{0}}=\left[\begin{array}{c}3.5 \\ 0\end{array}\right], R=1$, and $\mathbf{Q}=\left[\begin{array}{ll}0 & 0 \\ 0 & 1\end{array}\right]$. The coupling in this case can be shown to vanish for all values of $r$ and $k_{t}$, indicating that the problem is uncoupled and the artifact and controller can be designed sequentially. When the co-design problem is solved, no tradeoff is seen. For all values of $w_{a}$ and $w_{c}, f_{a}=81.53$ and $f_{c}=5.78$.

\section{MEMS Actuator Case Study}

The MEMS actuator considered here, shown in Fig. (5), was originally designed by Tung and Kurabayashi [42] and by Peters et al. [16]. The actuator utilizes four electrostatic comb-drive actuators to produce an out-ofplane displacement. To produce this displacement, each comb drive is excited with a voltage, $V$, resulting in horizontal (in-plane) movement $(\Delta X)$ of the silicon shuttles. The micro-hinges on the polydimethyl siloxane (PDMS) platform bend, and the platform moves vertically, or out-of-plane $(\Delta Z)$. The amount of movement resulting from the comb drives' actuation depends on both the applied voltage, $V$, and the physical dimensions of the actuator. Changing the actuator's dimensions results in a different output displacement for the same applied voltage.

The displacement of the actuator, $\Delta Z$, is given by

$$
\Delta Z=\left(h_{1}+h_{2}\right)(1-\cos \Delta \theta)+(t+p) \sin \Delta \theta
$$

where $p, t, h_{1}$, and $h_{2}$ are the hinge dimensions shown in Fig. (6), and $\Delta \theta$ is the angular displacement of the hinge.

The angular displacement $\Delta \theta$ satisfies the differential equation

$$
M_{M E M S} \Delta \ddot{\theta}+C_{M E M S} \Delta \dot{\theta}+K_{M E M S} \Delta \theta=A(\Delta \theta) V^{2}
$$




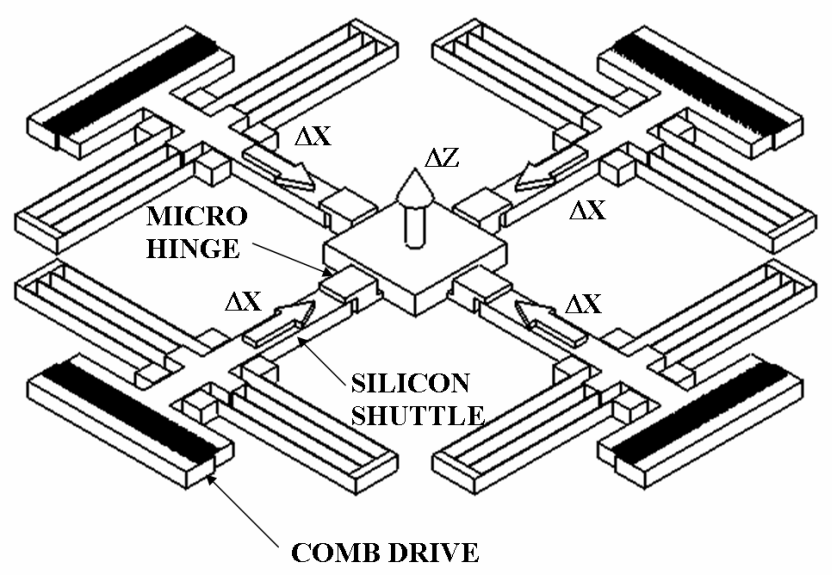

Figure 5: MEMS Actuator Configuration

where $M_{M E M S}, C_{M E M S}, K_{M E M S}$, and $A(\Delta \theta)$ are functions of the actuator geometry, as given in Eqs. (63) - (66) below. Derivations, and the equations for the masses and stiffnesses $M_{S i}, M_{P D M S}, M_{\text {hinge }}, K_{S i}$, and $K_{P D M S}$, are given in [42].

$$
\begin{aligned}
M_{M E M S}= & M_{S i}\left(h_{1}+h_{2}\right)^{2}+M_{P D M S}(t+p)^{2} \\
& +\frac{1}{3} M_{\text {hinge }}\left(\left(h_{1}+h_{2}\right)^{2}+(t+p)^{2}\right) \\
C_{M E M S}= & 2 \zeta \sqrt{\left(M_{S i}\left(h_{1}+h_{2}\right)^{2}+M_{P D M S}(t+p)^{2}\right)} \\
& \frac{\left(K_{S i}\left(h_{1}+h_{2}\right)^{2}+2 K_{P D M S}\right)}{K_{M E M S}=} \\
A(\Delta \theta)= & \frac{\left.K_{S i}\left(h_{1}+h_{2}\right)^{2}+2 h_{1}+h_{2}\right)}{d}\left(\left(h_{1}+h_{2}\right)-(t+p) \Delta \theta\right)
\end{aligned}
$$

where $\zeta=0.1$ is an experimentally determined parameter, $n=50$ is the number of fingers in the comb drive, $\epsilon_{o}=8.854 e-12 \mathrm{~F} / \mathrm{m}$ is the permittivity of vacuum, and $d=3 \mu \mathrm{m}$ is the width of a finger.

Alternatively, the system dynamics may be written in state-space form 


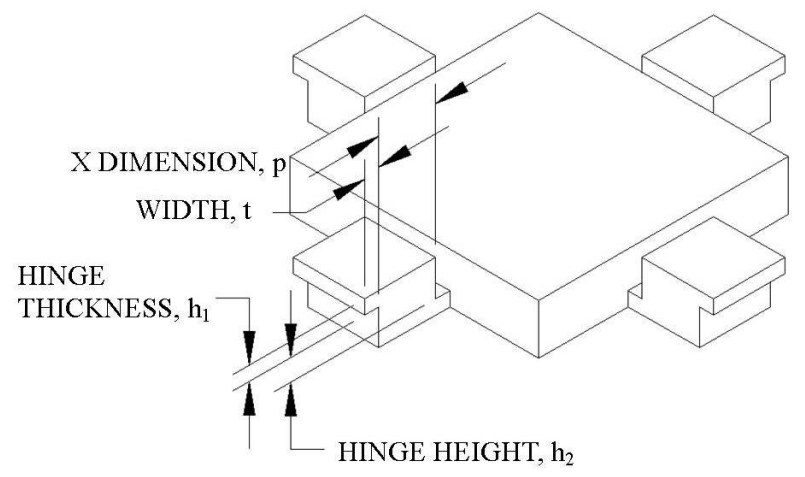

Figure 6: Micro-Hinge Structure

as

$$
\left[\begin{array}{c}
\Delta \dot{\theta} \\
\Delta \ddot{\theta}
\end{array}\right]=\left[\begin{array}{cc}
0 & 1 \\
-\frac{K_{M E M S}}{M_{M E M S}} & -\frac{C_{M E M S}}{M_{M E M S}}
\end{array}\right]\left[\begin{array}{c}
\Delta \theta \\
\Delta \dot{\theta}
\end{array}\right]+\left[\begin{array}{c}
0 \\
\frac{A(\Delta \theta)}{M_{M E M S}}
\end{array}\right] V^{2}
$$

Note that the coefficient $A$ in Eq. (62) is a function of $\Delta \theta$. Thus, the resulting controller design problem is non-linear. However, depending on the values of the various parameters, a linear approximation may be used. Naturally, such an approximation should be used cautiously in cases when the problem is highly non-linear; however, the linear assumption has been shown to be effective in previous analyses of this actuator [16, 25].

Two optimizations will be performed for this actuator. In the first optimization, the objective will be to maximize final displacement and minimize control effort for a given controller, subject to a set of constraints, and the relationship between coupling and controllability will be used to select design variables and choose an appropriate solution method. In the second optimization, the objective will be to maximize the steady-state displacement of the actuator and to minimize a LQR objective. A sequential optimization using a CPF will be performed, and the relationship between coupling and controllability will be used to select an appropriate CPF.

\subsection{Optimization for Final Displacement and Control Effort}

Assume that the MEMS actuator is to be optimized to maximize its final displacement, $\Delta Z_{f}$, and to minimize the control effort required to achieve 


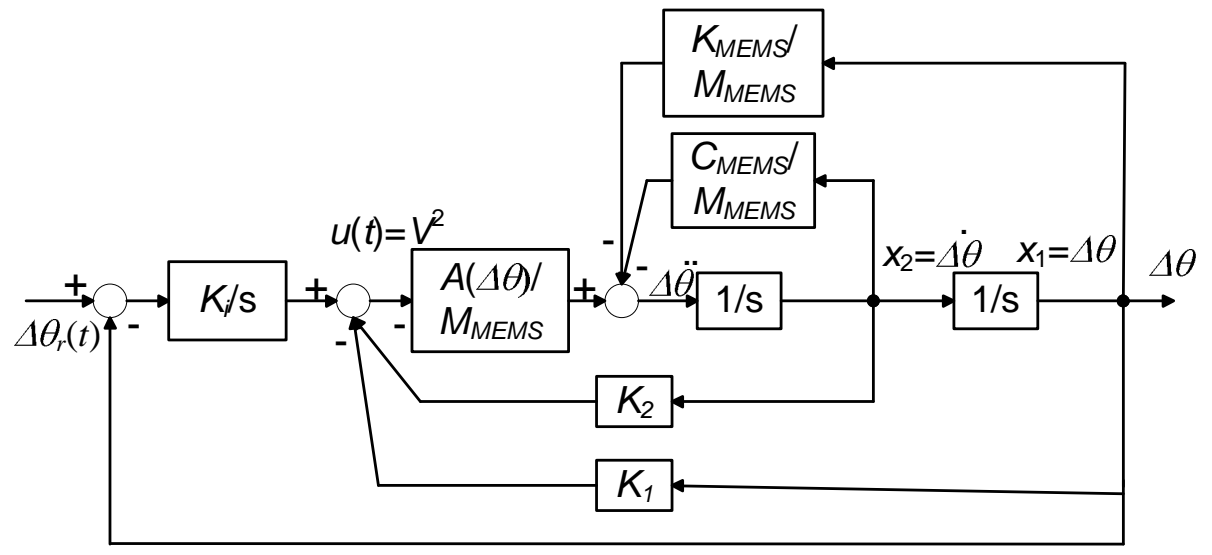

Figure 7: Control Architecture and System Dynamics

that displacement at a specified time, $t_{f}=0.25 \mathrm{~ms}$. The final displacement will be achieved at a $5 \%$ overshoot over the steady-state displacement.

An integral controller with state feedback is applied to the system, as shown in Fig. (7). It is assumed that the angle $\Delta \theta$ and the angular velocity $\Delta \dot{\theta}$ can be measured, and that the angle $\Delta \theta$ is to be controlled. The dynamics of the closed-loop system can then be written as

$$
\begin{aligned}
& M_{M E M S} \Delta \ddot{\theta}+\left(C_{M E M S}+K_{2} A(\Delta \theta)\right) \Delta \dot{\theta}+\left(K_{M E M S}+K_{1} A(\Delta \theta)\right) \Delta \theta \\
& -K_{i} A(\Delta \theta) \int_{0}^{t}\left(\Delta \theta_{r}-\Delta \theta\right) d \tau=0
\end{aligned}
$$

The artifact and control objective functions are given by the relations

$$
\begin{gathered}
f_{a}=-\Delta Z_{f}=-1.05 \Delta Z_{s s} \\
f_{c}=E=\int_{0}^{t_{f}}(u(t))^{2} d t=\int_{0}^{t_{f}}(V(t))^{4} d t
\end{gathered}
$$

with artifact inequality constraints, $\mathbf{g}_{\mathbf{a}}$, based on manufacturability, stress, and electrical and mechanical stability [25]. This optimization problem satisfies the description for Case I, where $R=1$, and thus the coupling can be evaluated using Eq. (30).

Five potential artifact design variables are considered, the hinge dimensions $p, t, h_{1}$, and $h_{2}$, and the shuttle length, $l_{1}$. It can be determined that there are no values for which the coupling vector will vanish, and thus a decoupled optimization problem cannot be formulated with them. However, 
Table 2: Nominal Values of Potential Design Variables

\begin{tabular}{c|c} 
Variable & Value \\
\hline$p(\mu \mathrm{m})$ & 80 \\
$t(\mu \mathrm{m})$ & 20 \\
$h_{1}(\mu \mathrm{m})$ & 20 \\
$h_{2}(\mu \mathrm{m})$ & 30 \\
$l_{1}(\mu \mathrm{m})$ & 700
\end{tabular}

it is useful to know how strongly coupled the problem would be with the potential design variables. Therefore, the derivatives of the controllability Grammian are evaluated numerically for the potential design variables at the nominal values given in Table 2. Note that the controllability Grammian does not depend on the potential design variable $l_{1}$, and therefore it can be stated that $l_{1}$ does not participate in the coupling.

$$
\begin{aligned}
\frac{\partial \mathbf{W}_{\mathbf{c}}}{\partial p} & =\left[\begin{array}{cc}
0 & -0.0002 \\
-0.0002 & -100.6
\end{array}\right] \\
\frac{\partial \mathbf{W}_{\mathbf{c}}}{\partial t} & =\left[\begin{array}{cc}
0 & -0.0002 \\
-0.0002 & -112.7
\end{array}\right] \\
\frac{\partial \mathbf{W}_{\mathbf{c}}}{\partial h_{1}} & =\left[\begin{array}{cc}
0 & -0.0038 \\
-0.0038 & -149.9
\end{array}\right] \\
\frac{\partial \mathbf{W}_{\mathbf{c}}}{\partial h_{2}} & =\left[\begin{array}{cc}
0 & 0.00001 \\
0.00001 & 61.84
\end{array}\right] \\
\frac{\partial \mathbf{W}_{\mathbf{c}}}{\partial l_{1}} & =\left[\begin{array}{ll}
0 & 0 \\
0 & 0
\end{array}\right]
\end{aligned}
$$

It can be seen that, in all these cases, the off-diagonal terms are quite small, and there is a zero term in each of them. The only term which is of any significant size is the lower diagonal term. This indicates that only one of the terms in the Grammian is significantly affected by the design variables, and it is the term that corresponds to the angular velocity of the actuator.

The variables chosen for this optimization are $l_{1}, p$, and $t$, while $h_{1}$ and $h_{2}$ are set as parameters. This choice is made, in the case of $h_{1}$, because of its relatively large effect on coupling, as seen from Eq. (73). In the case of $h_{2}$, while it has little effect on coupling, it also has a small effect on the artifact objective. The variables $p$ and $t$ have a comparatively large effect on 
the artifact objective. Since they do participate in coupling, a simultaneous optimization is chosen, and the optimization problem is given by

$$
\begin{aligned}
\begin{aligned}
\min _{\mathbf{d}_{\mathbf{a}}, \mathbf{d}_{\mathbf{c}}} \\
\text { subject to }
\end{aligned} & -w_{a} \Delta Z_{f}+w_{c} E \\
g_{a_{1}} & =t-5 h_{1} \leq 0 \\
g_{a_{2}} & =910-l_{1}-\frac{l_{p}}{2}-2 t+\frac{\Delta X_{s s}}{2} \leq 0 \\
g_{a_{3}} & =\frac{n \epsilon_{o}\left(h_{1}+h_{2}\right) V_{s s}^{2}}{d}-\frac{k_{b} \pi^{2} E_{P D M S} w\left(2 h_{1}+h_{2}\right)^{3}}{12 p^{2}} \leq(79) \\
g_{a_{4}} & =\Delta X_{s s}-\left(\frac{l_{S i}}{\sqrt{2}}-\frac{l_{f_{o}}}{2}\right) \leq 0 \\
g_{a_{5}} & =\frac{E_{P D M S} h_{1} \Delta \theta_{s s}}{2 p}-\sigma_{P D M S_{\text {max }}} \leq 0 \\
g_{a_{6}}= & \frac{3 \Delta X_{s s} E_{S i} b_{s}}{4 l_{S i}^{2}}-\sigma_{S i_{\text {max }}} \leq 0 \\
& 1 \mu \mathrm{m} \quad \leq p \leq 1000 \mu \mathrm{m} \\
& 1 \mu \mathrm{m} \quad \leq t \leq 1000 \mu \mathrm{m}
\end{aligned}
$$

where $\mathbf{d}_{\mathbf{a}}=\left(l_{1}, p, t\right)$ and $\mathbf{d}_{\mathbf{c}}=\left(K_{1}, K_{2}, K_{i}\right)$.

Solutions for various choices of $w_{a}$ and $w_{c}$ are shown in Fig. (8), and it can be seen that coupling is present, as expected.

Having a priori knowledge of the existence of coupling was useful in the formulation of this problem. Using this knowledge, it was possible to make an informed choice of artifact design variables. In this case, a completely decoupled problem could not be formulated unless the variables were restricted to $l_{1}$. However, knowing the relative contribution to coupling of each potential variable choice allows a designer to weigh the increased problem complexity posed by coupling against the potential improvement in the artifact objective function for that variable. In addition, given the knowledge that the problem was coupled, a simultaneous optimization was chosen. If the problem had been known to be uncoupled, then a sequential optimization would have been chosen instead.

\subsection{Optimization for Steady-State Displacement and LQR Control}

Assume that the MEMS actuator is to be optimized to maximize its steady-state displacement, $\Delta Z_{s s}$, and to minimize an LQR objective, in which 


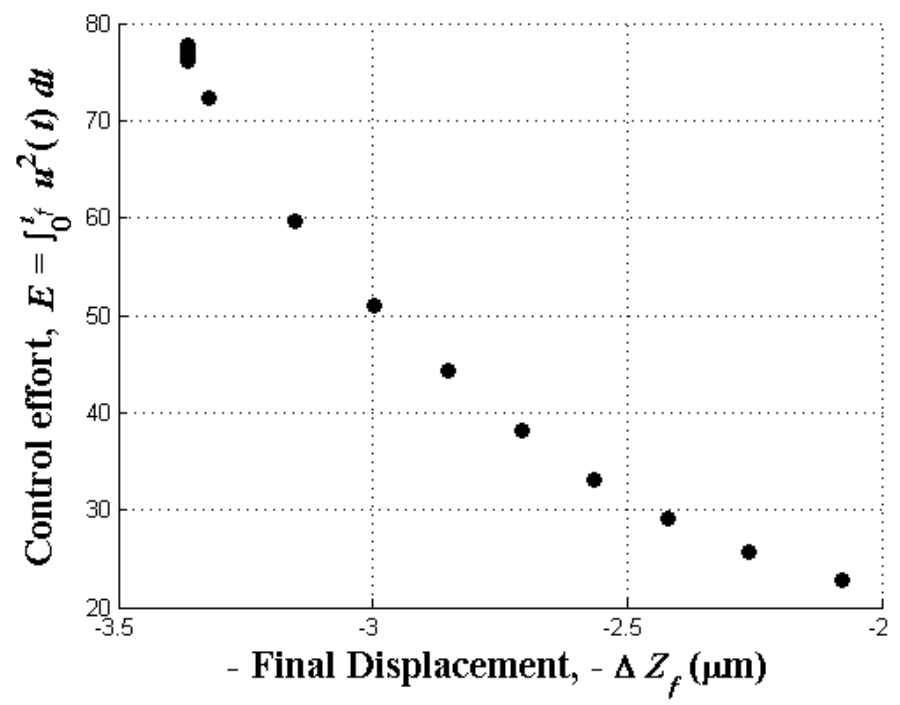

Figure 8: Optimal Solutions for MEMS Actuator

$R=1$ and $\mathbf{Q}=\mathbf{I}$. The variables chosen for this optimization are the same as those used in Section 5.1, i.e., $l_{1}, p$, and $t$, and the constraints are those given in Eqs. (77) - (82). The objective function is formulated as a linear weighted combination of the artifact objective function and the Control Proxy Function (CPF):

$$
\min _{\mathbf{d}_{\mathbf{a}}}-w_{1} \Delta Z_{s s}+w_{2} \chi
$$

where the steady-state displacement is given by

$$
Z_{s s}=\left(h_{1}+h_{2}\right)(1-\cos \Delta \theta)-(t+p) \sin \Delta \theta
$$

as derived in [42]. The LQR objective given by Eq. (51) is then minimized for the resultant artifact design. Obtaining an appropriate artifact design which is predisposed to effective LQR control depends on the CPF selected, and how well it captures the tradeoff between the artifact and controller design.

The relationship between coupling and controllability is used to select an appropriate $\mathrm{CPF}$. This optimization problem satisfies the criteria for Case III, and therefore it is known that the coupling is given by Eq. (59). This suggests that a CPF based on the steady-state controllability Grammian may be effective, so the CPF is chosen to be 


$$
\chi=\frac{1}{\operatorname{det}\left(\mathbf{W}_{\mathbf{c}}^{\infty}\right)}
$$

Solutions for various weights are shown in Fig. (9), with the solution set to the simultaneous optimization problem shown. It can be seen that this $\mathrm{CPF}$ is effective, and that the results of the modified sequential optimization are system-optimal. Knowing the relationship between coupling and controllability facilitated an appropriate choice of CPF, and the problem could have been solved without the need to formulate a simultaneous optimization.

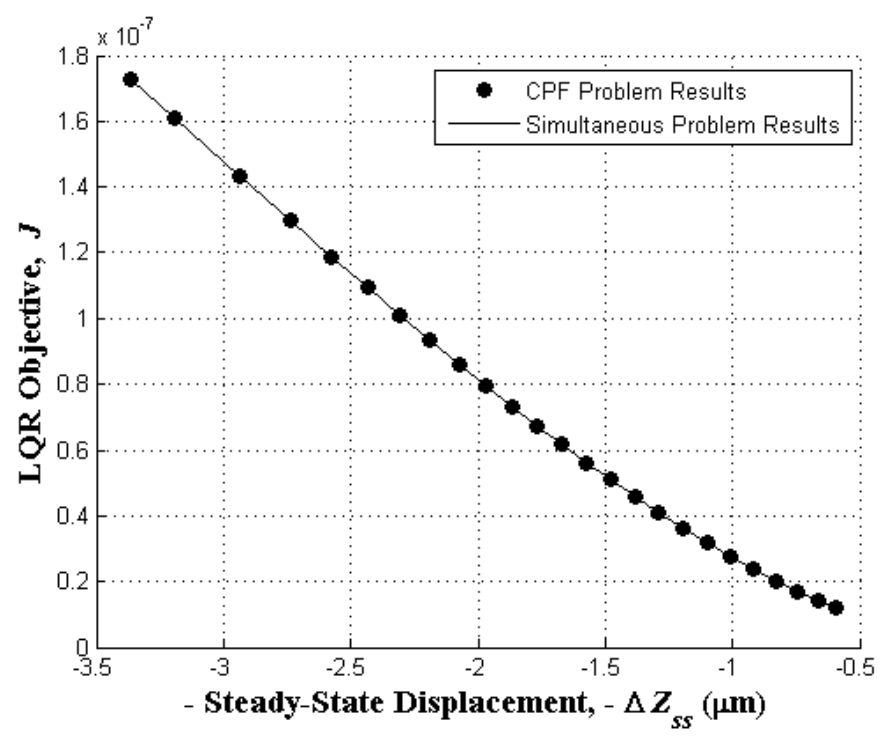

Figure 9: Optimal Solutions for MEMS Actuator with LQR Control

\section{Concluding Remarks}

In this paper, we have formulated an optimal co-design problem with a general controller objective that includes the accuracy of response, speed of response, and control effort required. We have shown that, for several important special cases of this formulation, it is possible to derive a relationship between the coupling vector and the controllability Grammian matrix for the system. This relation is independent of the artifact objective function and the controller architecture, and this allows the a priori calculation of 
coupling using the controllability Grammian. This relationship, while it requires knowledge of the control objective, does not require any knowledge of the control topology used to meet that objective.

While such a relationship for the general controller objective has not been derived, the cases presented here do give some important insight into this general problem. As an example, it is interesting to note that the first two cases, involving control effort and speed of response respectively, yield identical conditions for decoupling. This suggests that it is likely that these conditions will be valid for a controller objective that includes both of these two objectives; a proof of this conjecture is left for future work. It is further noted that the form of the LQR objective function is similar to the control objective in the first case, suggesting a strong fundamental connection among the various control objectives.

These results are significant for their use in understanding the nature of the trade-off between design and control, including a better picture of what the complete Pareto curve might look like. Use of coupling information to characterize the Pareto curve can be useful in cases where obtaining a large number of Pareto optimal solutions is expensive, as discussed in [43, 44]. These results are also useful in problem formulation and choice of a solution method.

When a co-design problem is being formulated and artifact design variables are being chosen, the strength of the coupling in a potential formulation can be considered. In the case of LQR problems, it may be possible to choose the matrices $\mathbf{Q}$ and $\mathbf{R}$ in order to facilitate problem decoupling. In addition, for all cases presented in this paper, the effect on coupling of potential artifact design variables can be considered. If a potential design variable participates in coupling, then its effect on the artifact objective function can be evaluated to determine whether it is significant enough to justify the increased problem complexity. If a variable participates in coupling but has a relatively small effect on the artifact objective function, it may be chosen as a parameter, resulting in an uncoupled problem which can be solved sequentially with no loss of optimality.

If it is determined that a problem cannot be decoupled, or if the potential improvement in the artifact objective function justifies the increased complexity of a coupled problem, then an appropriate solution method can be chosen. It is known, in this case, that a sequential solution method will not guarantee optimality, and a method that accounts for coupling should be used. Such methods include simultaneous problem formulations, meth- 
ods involving decomposition and coordination of sub-systems, and the use of a Control Proxy Function (CPF) [25, 26, 27]. The a priori knowledge of coupling is particularly useful in the CPF method, since it provides a basis for choosing an appropriate CPF. Several specific CPFs have been derived, based on this understanding of coupling, as described in [28]. Such methods may also be useful in problems with bi-directional coupling, such as the combined active/passive suspension presented in [28]. Further work will show when this is the case, and may broaden the range of problems for which this type of approach is appropriate.

Future work may include the extension of this work to cover other controller objectives that can be formulated as specific cases of the general form given here. This controller objective could also be expanded to broaden its generality, by including the Mayer (terminal cost) term and other possible objectives, such as energy-based terms. Physical conditions and specific types of mechanical elements that might exhibit decoupling also merit investigation, as well as the application of this work to develop additional CPFs. It is also important to note that this analysis is limited exclusively to linear systems, and in particular to linear time invariant systems. While many systems fall into this category, and others can be approximated in this fashion, it is a significant limitation. Future work should also include extensions to

time-varying linear systems, and to nonlinear systems, in order to expand the applicability of this work.

\section{Acknowledgments}

This work was supported in part by NSF grant \#0625060 and by the Automotive Research Center (ARC). This support is gratefully acknowledged.

\section{References}

[1] R. Haftka, Integrated structure/control optimization of space structures, in: Proceedings of the AIAA Dynamics Specialists Conference, AIAA, Long Beach, CA, 1990, pp. 1-9.

[2] R. Kosut, G. Kabuli, S. Morrison, Y. Harn, Simultaneous control and structure design for large space structures, in: Proceedings of the American Control Conference, IEEE, San Diego, CA, 1990, pp. 860-865. 
[3] F. Khorrami, J. Rastegar, Simultaneous structure and control design for a flexible pointing system actuated by active materials, in: Proceedings of the 34th Conference on Decision \& Control, New Orleans, LA, 1995.

[4] R. Isermann, On the design and control of mechatronic systems - a survey, IEEE Transactions on Industrial Electronics 43 (1996) 4-15.

[5] C. Chen, C. Cheng, Integrated design for a mechatronic feed drive system of machine tools, in: IEEE/ASME International Conference on Advanced Intelligent Mechatronics, Monterey, CA, 2005.

[6] E. Akdoğan, M. A. Adli, The design and control of a therapeutic exercise robot for lower limb rehabilitation: Physiotherabot, Mechatronics 21 (2011) 509-522.

[7] M. G. Villarreal-Cervantes, C. A. Cruz-Villar, J. Alvarez-Gallegos, E. A. Portilla-Flores, Robust structure-control design approach for mechatronic systems, Mechatronics, IEEE/ASME Transactions on 18 (5) (2013) 1592-1601.

[8] J. Wan, Q. Li, F. Wu, Integrated design and control of a closed-chain mechanism, in: Proceedings of the Seventh International Conference on Control, Automation, Robotics and Vision, IEEE, Singapore, 2002, pp. 1349-1353.

[9] T. Ravichandran, D. Wang, G. Heppler, Simultaneous plant-controller design optimization of a two-link planar manipulator, Mechatronics 16 (2006) 233-242.

[10] J. M. Rieber, D. G. Taylor, Integrated control system and mechanical design of a compliant two-axes mechanism, Mechatronics 14 (2004) 1069-1087.

[11] J. M. Rodriguez-Fortun, J. Orus, J. Alfonso, J. R. Sierra, F. Buil, F. Rotella, J. Castellanos, Model-based mechanical and control design of a three-axis platform, Mechatronics 22 (2012) 958-969.

[12] P. Ouyang, Q. Li, W. J. Zhang, L. Guo, Design, modeling and control of a hybrid machine system, Mechatronics 14 (2004) 1197-1217. 
[13] J. S. Rao, R. Tiwari, Design optimization of double-acting hybrid magnetic thrust bearings with control integration using multi-objective evolutionary algorithm, Mechatronics 19 (2009) 945-964.

[14] H. Wolfram, R. Schmiedel, K. Hiller, T. Aurich, W. Gunther, S. Kurth, J. Mehner, W. Dotzel, T. Gebner, Model building, control design and practical implementation of a high precision, high dynamical MEMS acceleration sensor, in: Society of Photo-Optical Instrumentation Engineers, SPIE, Sevilla, Spain, 2005, pp. 1-15.

[15] K. Oldham, X. Huang, A. Chahwan, R. Horowitz, Design, fabrication and control of a high-aspect ratio microactuator for vibration suppression in a hard disk drive, in: Proceedings of the IFAC World Congress, Prague, 2005.

[16] D. L. Peters, K. Kurabayashi, P. Y. Papalambros, A. G. Ulsoy, Co-design of a MEMS actuator and its controller using frequency constraints, in: Proceedings of the ASME Dynamic Systems and Control Conferences, ASME, Ann Arbor, MI, 2008, paper number DSCC 2008-2212.

[17] V. S. Shabde, K. A. Hoo, Quantifying the effects of process design on controllability and operability, in: AIChE Annual Meeting, San Francisco, CA, 2006.

[18] V. S. Shabde, K. A. Hoo, Optimum controller design for a spray drying process, Control Engineering Practice 16 (2008) 541-552.

[19] R. Citalan-Lara, C. A. Cruz-Villar, Multidisciplinary optimization of servodrives for robot manipulators, in: 2014 IEEE/ASME International Conference on Advanced Intelligent Mechatronics, IEEE/ASME, Besancon, France, 2014, pp. 38-43.

[20] W. Gao, Y. Zou, F. Sun, Optimal component sizing for a parallel hybrid bus based on dynamic programming, in: Transportation Electrification Asia-Pacific (ITEC Asia-Pacific), 2014 IEEE Conference and Expo, IEEE, 2014, pp. 1-5.

[21] J. T. Allison, T. Guo, Z. Han, Co-design of an active suspension using simultaneous dynamic optimization, ASME Journal of Mechanical Design 136 (8). 
[22] K. Youcef-Toumi, Modeling, design, and control integration: A necessary step in mechatronics, IEEE/ASME Transactions on Mechatronics 1 (1996) 29-38.

[23] R. Patil, Z. Filipi, H. Fathy, Computationally efficient combined plant design and controller optimization using a coupling measure, ASME Journal of Mechanical Design 134 (7).

[24] H. Fathy, Combined Plant and Control Optimization: Theory, Strategies and Applications, PhD Thesis, University of Michigan, Ann Arbor, MI (April 2003).

[25] D. L. Peters, Coupling and Controllability in Optimal Design and Control, PhD Thesis, University of Michigan, Ann Arbor, MI (April 2010).

[26] D. L. Peters, P. Y. Papalambros, A. G. Ulsoy, Sequential co-design of an artifact and its controller via control proxy functions, in: Proceedings of the 5th IFAC Symposium on Mechatronic Systems, IFAC, Cambridge, MA, 2010.

[27] D. L. Peters, P. Y. Papalambros, A. G. Ulsoy, Control proxy functions for sequential design and control optimization, ASME Journal of Mechanical Design 133 (9).

[28] D. L. Peters, P. Y. Papalambros, A. G. Ulsoy, Sequential co-design of an artifact and its controller via control proxy functions, Mechatronics 23 (2013) 409-418.

[29] J. T. Allison, Plant-limited co-design of an energy-efficient counterbalanced robotic manipulator, ASME Journal of Mechanical Design 135 (10).

[30] Y. Jiang, Y. Wang, S. A. Bortoff, Z. Jiang, Optimal codesign of nonlinear control systems based on a modified policy iteration method, Neural Networks and Learning Systems, IEEE Transactions on 26 (2) (2015) 409-414.

[31] Y. Wang, S. A. Bortoff, Co-design of nonlinear control systems with bounded control inputs, Mitsubishi Electric Research Laboratories. 
[32] J. T. Allison, S. Nazari, Combined plant and controller design using decomposition-based design optimization and the minimum principle, in: Proceedings of the ASME 2010 International Design Engineering Technical Conferences \& Computers and Information in Engineering Conference, ASME, Montreal, Canada, 2010, paper Number DETC2010-28887.

[33] H. Fathy, P. Y. Papalambros, A. G. Ulsoy, On combined plant and control optimization, in: 8th Cairo University International Conference on Mechanical Design and Production, Cairo University, Cairo, Egypt, 2004.

[34] J. Reyer, H. Fathy, P. Y. Papalambros, A. G. Ulsoy, Comparison of combined embodiment design and control optimization strategies using optimality conditions, in: Proceedings of the ASME Design Engineering Technical Conference and Computers and Information in Engineering Conference, ASME, Pittsburgh, PA, 2001.

[35] S. F. Alyaqout, P. Y. Papalambros, A. G. Ulsoy, Coupling in design and robust control optimization, in: Proceedings of the European Control Conference, Kos, Greece, 2007.

[36] C. Bloebaum, Coupling strength-based system reduction for complex engineering design, Structural Optimization 10 (1995) 113-121.

[37] D. L. Peters, P. Y. Papalambros, A. G. Ulsoy, On measures of coupling between the artifact and controller optimal design problems, in: Proceedings of the ASME Design Engineering Technical Conference \& Computers in Engineering Conference, ASME, San Diego, CA, 2009, paper number DETC 2009-86868.

[38] S. Skogestad, I. Postlethwaite, Multivariable Feedback Control: Analysis and Design, John Wiley and Sons, Ltd., West Sussex, UK, 2005.

[39] A. Bryson, Y. Ho, Applied Optimal Control, Hemisphere Publishing Corporation, New York, NY, 1975.

[40] P. Papalambros, D. Wilde, Principles of Optimal Design: Modeling and Computation, Cambridge University Press, Cambridge, NY, 2000. 
[41] D. L. Peters, P. Y. Papalambros, A. G. Ulsoy, Relationship between coupling and the controllability Grammian in co-design problems, in: Proceedings of the American Control Conference, ASME, Baltimore, MD, 2010.

[42] Y. Tung, K. Kurabayashi, A single-layer PDMS-on-silicon hybrid microactuator with multi-axis out-of-plane motion capabilities - part I: Design and analysis, Journal of Microelectromechanical Systems 14 (2005) $548-557$.

[43] B. D. Frischknecht, D. L. Peters, P. Y. Papalambros, Pareto set analysis: Local measures of objective coupling in multiobjective design optimization, in: 8th World Congress on Structural and Multidisciplinary Optimization, Lisbon, Portugal, 2009.

[44] B. D. Frischknecht, D. L. Peters, P. Y. Papalambros, Pareto set analysis: Local measures of objective coupling in multiobjective design optimization, Structural \& Multidisciplinary Optimization 43 (5) (2011) 617-630. 